\title{
WaveLight ${ }^{\circledR}$ Contoura topography-guided planning: contribution of anterior corneal higher-order aberrations and posterior corneal astigmatism to manifest refractive astigmatism
}

This article was published in the following Dove Press journal:

Clinical Ophthalmology

\author{
Avi Wallerstein ${ }^{1,2}$ \\ Mathieu Gauvin ${ }^{1,2}$ \\ Mark Cohen ${ }^{2,3}$ \\ 'Department of Ophthalmology, \\ Faculty of Medicine, McGill University, \\ Montreal, QC, Canada; ${ }^{2}$ LASIK MD, \\ Montreal, QC, Canada; ${ }^{3}$ Department \\ of Surgery, Faculty of Medicine \\ and Health Sciences, University of \\ Sherbrooke, Sherbrooke, QC, Canada
}

Clinical refractive astigmatism (RA) and topography-measured anterior corneal astigmatism (ACA) are rarely identical in magnitude and axis. ${ }^{1}$ This difference, when expressed vectorially, is termed ocular residual astigmatism (ORA). ${ }^{1}$ What accounts for this difference remains a topic that needs further research, and has implications as to what refraction to treat with topography-guided excimer laser ablations in order to maximize outcomes. ${ }^{1}$ This research letter investigates aspects of two contributing factors: posterior corneal astigmatism (PCA) and anterior corneal higher-order aberrations (CHOAs).

We compared RA to ACA magnitude in 5,403 eyes to reveal that $87 \%$ with-the-rule (WTR) corneal cylinder eyes had less RA magnitude than ACA magnitude, and $89 \%$ against-the-rule (ATR) corneal cylinder eyes had RA magnitude greater than ACA magnitude (Figure 1). Published data show that $87 \%$ of corneas have ATR posterior corneal astigmatism, ${ }^{5}$ which, if additive to ACA, could explain the decreased RA seen in most WTR corneal cylinder eyes, and the increased RA in most ATR corneal cylinder eyes (Figure 1). Furthermore, our data show that the ACA axis location correlates to the amount of difference between RA and ACA (Figure 1), supporting the notion that the interaction between ACA and PCA impacts RA by modifying the total corneal astigmatism.

A recent paper $^{2}$ claimed that PCA does not contribute significantly to RA, while other publications have reported that PCA compensates between $22 \%$ and $31 \%$ of the ACA. ${ }^{3,4}$ A large cohort study revealed that PCA accounts for more than $-0.5 \mathrm{D}$ in $9 \%$ of eyes. ${ }^{5}$ This magnitude of PCA cannot be taken as clinically negligible, particularly for eyes with a greater amount of cylinder. It is also why PCA is now used for intra-ocular lens surgical planning, with improved outcomes. ${ }^{6}$ With topographyguided treatments, only treating the Contoura-measured ACA could allow the PCA to manifest as RA post-operatively, as can be seen with TORIC IOL surgery that ignores PCA. ${ }^{6}$

A recent series examined six eyes with significant ORA and theorized that anterior CHOAs were the cause of the discrepancy between RA and ACA. ${ }^{2}$ For illustrative purposes, the author characterized CHOAs as creating an ovalization of the central cornea, interacting with an ovalization produced by ACA. ${ }^{2}$ Depending on how these two ovals are aligned, the net RA will summate or negate. ${ }^{2}$ To objectively investigate
Correspondence: Avi Wallerstein LASIK MD, Montreal, I 250 Rene-Levesque Blvd W, Montreal, QC H3B 4W8, Canada Tel + I 5149089888 Ext 2316

Email awallerstein@lasikmd.com 


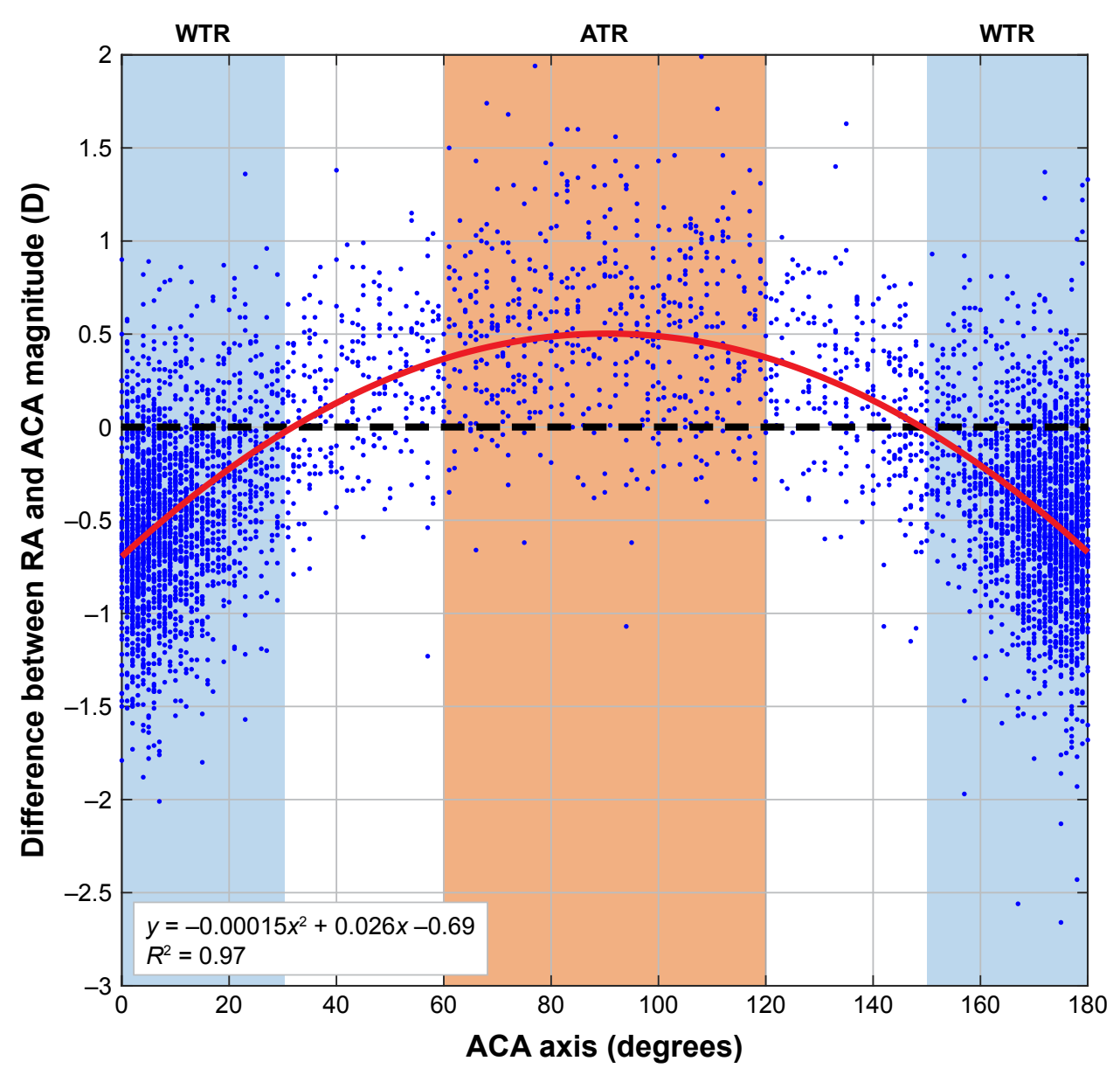

Figure I Difference between RA and ACA magnitude (ie, RA magnitude - ACA magnitude) in relation to the ACA axis and quadratic fitting of this relationship (red curve). Data points (blue dots) above the black dashed line indicate eyes where the ACA is smaller than the RA magnitude, and below show eyes where the ACA is greater. Blue-shaded area: WTR-ACA eyes; red-shaded area: ATR-ACA eyes.

Abbreviations: ACA, anterior corneal astigmatism; ATR, against-the-rule; RA, refractive astigmatism; WTR, with-the-rule.

this claim, we developed an advanced algorithm that analyzed the Contoura (WaveLight ${ }^{\circledR}$ EX-500; Alcon Laboratories, Inc., Fort Worth, TX, USA) CHOA ablation profile in 3D, accurately detailing the CHOA ellipse (Figure 2A). We then studied the vectorial relationship between the calculated CHOAs and ACA ellipses in 12 eyes (Figure 2B). While we found six of 12 cases that supported the summation notion, we also found six contradictory cases, and could therefore not validate this theory (Figure 2B). This was illustrated in cases $2,6,9$, and 12 , where ellipses with similar orientations should have summated and increased the RA, but did not. For case 11, the ellipses with perpendicular orientation should have partially canceled each other, but did not. Case 1 is an example that could not be fitted by an ellipse as the pattern was circular. While we agree that CHOAs also affect RA, a concept previously reported by Zhou et al, ${ }^{7}$ the claim that ORA is mainly accounted for by anterior CHOAs in the large majority of eyes ${ }^{2}$ is not supported by the current research letter's findings or by previous litterature..$^{1,3-6}$

The difference between RA and ACA is thought to be attributed to several factors, including anterior CHOAs, PCA, anterior and posterior lenticular astigmatism, refractive index variability, decentration of the crystalline lens, as well as from retinal tilt and cortical perception. ${ }^{1}$ Contoura topographyguided software determines the anterior CHOAs and the true ACA from a high resolution Placido disc topographer. The Contoura-measured ACA does not consider the manifest RA and does not supply information on how anterior CHOAs influence RA. RA encompasses all sources of astigmatism, from the anterior cornea to the brain. Since Contoura treatments partially remove anterior $\mathrm{CHOAs}$, to produce a more accurate nomogram for topography-guided ablations, one would need to fully elucidate - possibly using ray tracing technology - the contribution of anterior CHOAs to manifest RA, as well as the effects of PCA and the remaining internal optics. 
A

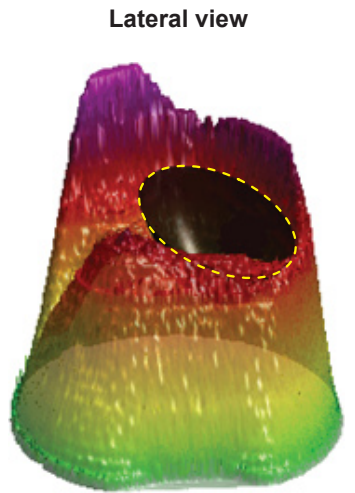

Bottom view

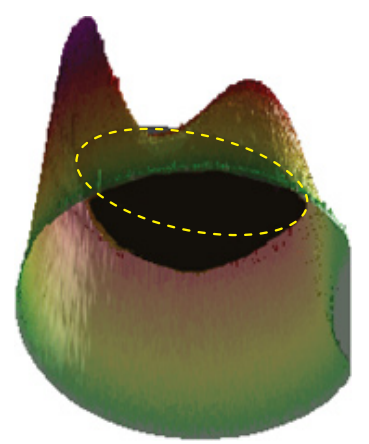

Top view

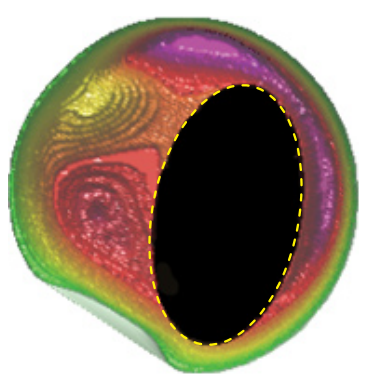

B

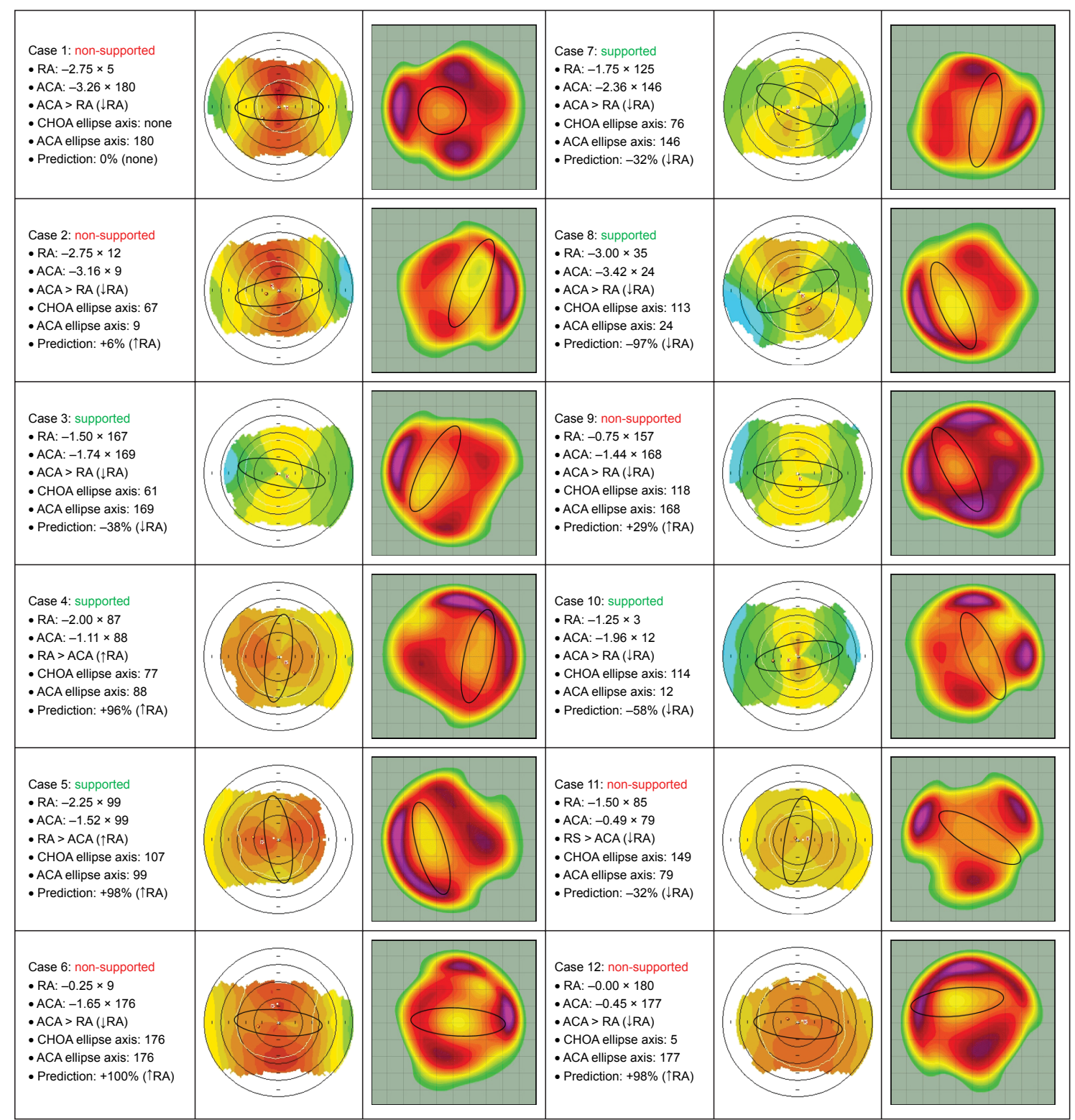

Figure 2 (A) Objective algorithm-based identification of a 3D ellipsoid in a representative Contoura CHOA ablation profile visualized at different angles. The CHOAs' ellipse axis was calculated using the top view. (B) Case series of 12 eyes used to test Motwani's claim. Left column, eye parameters; middle column, topographical corneal astigmatism where the ovalization is indicated by a black ellipse; right column, CHOAs' profile where the ovalization is indicated by a black ellipse as in Motwani's work. ${ }^{2}$ Vectorial analyses were used to predict the effect of the interacting ellipses on RA as a positive or negative percentage change, setting both ellipses at equal magnitude. "Supported" and "Non-Supported" identify cases where Motwani's claim is validated or not, respectively.

Abbreviations: ACA, anterior corneal astigmatism; RA, refractive astigmatism; CHOAs, corneal higher-order aberrations. 


\section{Disclosure}

The authors report no conflicts of interest in this work.

\section{References}

1. Alpins N. Practical Astigmatism Planning and Analysis. SLACK Incorporated; Thorofare, NJ, USA. 2017.

2. Motwani M. The use of WaveLight ${ }^{\mathbb{1}}$ Contoura to create a uniform cornea: the LYRA Protocol. Part 1: the effect of higher-order corneal aberrations on refractive astigmatism. Clin Ophthalmol. 2017;11:897-905.

3. Atchison DA, Markwell EL, Kasthurirangan S, Pope JM, Smith G, Swann PG. Age-related changes in optical and biometric characteristics of emmetropic eyes. J Vis. 2008;829(4):29.1-20.
4. Dubbelman M, Sicam VA, van der Heijde GL. The shape of the anterior and posterior surface of the aging human cornea. Vision Res. 2006; 46(6-7):993-1001

5. Koch DD, Ali SF, Weikert MP, Shirayama M, Jenkins R, Wang L. Contribution of posterior corneal astigmatism to total corneal astigmatism. $J$ Cataract Refract Surg. 2012;38(12):2080-2087.

6. Reitblat O, Levy A, Kleinmann G, Abulafia A, Assia EI. Effect of posterior corneal astigmatism on power calculation and alignment of toric intraocular lenses: Comparison of methodologies. $J$ Cataract Refract Surg. 2016;42(2):217-225.

7. Zhou W, Stojanovic A, Utheim TP. Assessment of refractive astigmatism and simulated therapeutic refractive surgery strategies in coma-likeaberrations-dominant corneal optics. Eye Vis. 2016;3:13.
Clinical Ophthalmology

\section{Publish your work in this journal}

Clinical Ophthalmology is an international, peer-reviewed journal covering all subspecialties within ophthalmology. Key topics include: Optometry; Visual science; Pharmacology and drug therapy in eye diseases; Basic Sciences; Primary and Secondary eye care; Patient Safety and Quality of Care Improvements. This journal is indexed on

\section{Dovepress}

PubMed Central and CAS, and is the official journal of The Society of Clinical Ophthalmology (SCO). The manuscript management system is completely online and includes a very quick and fair peer-review system, which is all easy to use. Visit http://www.dovepress.com/ testimonials.php to read real quotes from published authors. 